\title{
G somentem \\ Diastolic Dysfunction and Impaired Cardiac Output Reserve in Dysmetabolic Nonhuman Primate With Proteinuria
}

\section{Johannes Wikstrom}

Bioscience, Research and Early Development, Cardiovascular, Renal and Metabolism, BioPharmaceuticals R\&D, AstraZeneca, Gothenburg, Sweden

\section{Yongqiang Liu}

Crown Bioscience Inc., 6 West Beijing Road, Taicang, Jiangsu, China

\section{Carl Whatling}

Translational Science and Experimental Medicine, Research and Early Development, Cardiovascular, Renal and Metabolism, BioPharmaceuticals R\&D, AstraZeneca, Gothenburg, Sweden

\section{Li-ming Gan}

Early Clinical Development, Research and Early Development, Cardiovascular, Renal and Metabolism, BioPharmaceuticals R\&D, AstraZeneca, Gothenburg, Sweden

\section{Peter Konings}

Quantitative Biology, Discovery Sciences, R\&D, AstraZeneca, Gothenburg, Sweden

\section{Binchen Mao}

Crown Bioscience Inc., 6 West Beijing Road, Taicang, Jiangsu, China

\section{Chao Zhang}

Crown Bioscience Inc., 6 West Beijing Road, Taicang, Jiangsu, China

\section{Yanqin Ji}

Crown Bioscience Inc., 6 West Beijing Road, Taicang, Jiangsu, China

\section{Yong-Fu Xiao}

Crown Bioscience Inc., 6 West Beijing Road, Taicang, Jiangsu, China

\section{Yixin Wang ( $\nabla$ yxwang2000@gmail.com )}

Crown Bioscience Inc., 6 West Beijing Road, Taicang, Jiangsu, China https://orcid.org/0000-00023466-429X

\section{Research}

Keywords: Diabetic complications, Cardiorenal dysfunction, Metabolic syndrome, Monkey model, Dobutamine tress test, echocardiography

Posted Date: September 28th, 2020 
DOI: https://doi.org/10.21203/rs.3.rs-80071/v1

License: (c) (1) This work is licensed under a Creative Commons Attribution 4.0 International License. Read Full License

Version of Record: A version of this preprint was published at Journal of Diabetes and its Complications on April 1st, 2021. See the published version at https://doi.org/10.1016/j.jdiacomp.2021.107881. 


\section{Abstract}

\section{Background}

Cardiorenal complications are common in patients with dysmetabolism and diabetes. The present study aimed to examine if a nonhuman primate (NHP) model with spontaneously developed metabolic disorder and diabetes develops similar complications to humans, such as proteinuria and cardiac dysfunction at resting condition or diminished cardiac functional reserve following dobutamine stress echocardiography (DSE).

\section{Methods and Results}

A total of 66 dysmetabolic and diabetic cynomolgus (Macaca fascicularis) NHPs were enrolled to select 19 NHPs (MetS) with marked metabolic disorders and diabetes (fasting blood glucose: $178 \pm 18$ vs. $61 \pm 3$ $\mathrm{mg} / \mathrm{dL}$ ) accompanied by proteinuria (ACR: $134 \pm 34 \mathrm{vs}$. $1.5 \pm 0.4 \mathrm{mg} / \mathrm{mmol}$ ) compared to 8 normal NHPs (CTRL). Under resting condition, MetS NHPs showed mild left ventricular (LV) diastolic dysfunction (E/A: $1 \pm 0.06$ vs. $1.5 \pm 0.13$ ), but with preserved ejection fraction (EF: $65 \pm 2$ vs. $71 \pm 3 \%$ ) compared to CTRL. DSE with an intravenous infusion of dobutamine at ascending doses $(5,10,20,30$ and $40 \mu \mathrm{g} / \mathrm{kg} / \mathrm{min}, 7 \mathrm{~min}$ for each dose) resulted in a dose-dependent increase in cardiac function, however, with a significantly diminished magnitude at the highest dose of dobutamine infusion $(40 \mu \mathrm{g} / \mathrm{kg} / \mathrm{min})$ in both diastole (E/A: $-12 \pm 3$ vs. $-38 \pm 5 \%$ ) and systole (EF: $25 \pm 3$ vs. $33 \pm 5 \%$ ) as well as $\sim 42 \%$ reduced cardiac output reserve (COR: $63 \pm 8$ vs. $105 \pm 18 \%, p<0.02$ ) in the MetS compared to CTRL NHPs.

\section{Conclusion}

These data demonstrate that MetS NHPs with cardiorenal complications: proteinuria, LV diastolic dysfunction and preserved LV systolic function under resting conditions displayed compromised cardiac functional reserve under dobutamine stress. Based on these phenotypes, this NHP model of diabetes with cardiorenal complications can be used as a highly translational model mimic human disease for pharmaceutical research.

\section{Background}

Echocardiography is commonly used in the clinic as an essential diagnostic tool to noninvasively examine parameters such as cardiac structure, ventricular function and non-perfused myocardium since it is convenient, rapid, economical, and radiation free [1, 2]. Among the echocardiography indices, ejection fraction (EF) represents a marker of left ventricular (LV) systolic function, while the ratio of LV early (LVE) and late (LVA) trans-mitral Doppler inflow velocity (E/A) is a marker of LV diastolic function, both of which are commonly used to evaluate LV function in risk assessment of cardiac death in patients with heart failure. It is well known that EF is a major determinant of long-term survival in patients with coronary artery disease [3]. Doppler flow propagation velocity has also been proposed to assess LV compliance and the severity of diastolic dysfunction [4, 5]. 
In some patients with heart disease at early stage, cardiac dysfunction may be mild and difficult to detect under resting condition [6-8]. Stress tests, such as treadmill exercise, can be used to assess cardiac functional reserve by pushing the heart to work more intensively. However, since not every patient is able to exercise at an adequate intensity (heart rate response), therefore, a pharmacological stress test is often used to assess cardiac functional reserve [6-8]. Among the pharmacological stress agents with positive inotropic and chronotropic effects, dobutamine stress echocardiography (DSE), a reliable cardiac risk stratify, is commonly used in the clinic because of its excellent safety profile, clinical accuracy and cost effectiveness, with clinical results superior to regular exercise electrocardiography [6-8]. Dobutamine is an adrenergic receptor (AR) agonist with its b1 activity mediating the inotropic effect to increase LV contractility and 1 activity mediating the chronotropic effects to increase heart rate (HR), both of which enhance the heart functional performance by increasing cardiac output (CO). Thus, DSE is commonly used in the clinic to examine the cardiac functional reserve in patients with mild cardiac dysfunction in the early stages of disease progression that may not be detectable under resting conditions. Clinical studies are now available refining the common use of DSE preoperatively in patients with valvar disease, as well as in emergency cardiac situations [9-11]. Recently available long-term results from several independent clinical trials, combined with image enhancements, have improved the ability to detect signs of subtle coronary artery disease and determine myocardial viability $[12,13]$.

Nonhuman primate (NHP) models of spontaneously developed obesity, dysmetabolism and diabetes are used as the most translatable animal model in basic research to investigate the pathophysiological mechanisms, as well as pharmaceutical testing of novel therapies [14-16]. Similar to human patients, dysmetabolic and diabetic NHPs also develop complications such as nephropathy [17] , nonalcoholic fatty liver disease (NAFLD) / steatohepatitis (NASH) [18], and cardiac dysfunction [19]. In a previous study, we reported that when using noninvasive echocardiography, dysmetabolic and diabetic NHPs also presented mild cardiac dysfunctions under resting conditions [19]. However, in some NHPs in the early stages of disease progression, cardiac function may still be normal or only present mild changes under resting conditions that might not be easily detectable, thus, the stress test may be able to detect the subtle changes in diminished cardiac functional reserve. However, the exercise stress test is not practical in preclinical studies in animal models, therefore, pharmacological stress tests are the most practical choice. Indeed, Wang et al. reported that resting cardiac function was preserved in atherosclerotic apolipoprotein E deficient mice [20] and hyperglycemic minipigs [21] at early stages of disease; however, following the pharmacological stress test, cardiac functional reserve was detected to be significantly diminished in both models.

The aim of the present study was to use DSE to detect if the cardiac functional reserve is compromised in a NHP model of spontaneously developed dysmetabolism, diabetes and proteinuria with subtle or mild cardiac dysfunction that may not be detectable at resting conditions in the early stages of disease progression.

\section{Methods}


Animal care and use were conducted in accordance with applicable AAALAC regulations and guidelines. All experimental protocols and procedures were approved by Crown Bioscience's institutional animal care and use committee (IACUC).

The NHPs were individually housed at Crown Bioscience Inc., Taicang, China in species appropriate cages, kept in temperature-controlled rooms maintained at $23 \pm 3^{\circ} \mathrm{C}$ on a 12-hour light-dark cycle, with free access to water, twice daily feeding with a nutritionally balanced normal primate chow (Beijing Keao Xieli Feed Co., LTD, Beijing, China) containing $~ 52 \%$ carbohydrate, $\geq 16 \%$ crude protein, $\geq 4 \%$ crude fat, $\leq 7 \%$ ash, $\leq 4 \%$ fiber, $0.8 \sim 1.2 \%$ calcium, and $\leq 10 \%$ moisture, enriched with seasonal fruits and vegetables. All animals were weight stable during the study and monitored closely for mobility, feces and any other abnormal behavior and activities.

A cohort of 60 male and female cynomolgus monkeys (Macaca fascicularis) with various degrees of dysmetabolism were screened with measurements of blood and urine chemistry and echocardiogram. From this screen, $20 \mathrm{NHPs}$ with marked dysmetabolic profiles and proteinuria (MetS) were selected for DSE along with 8 normal NHPs as a control group (CTRL).

Blood and urine sample collection and bioanalysis

Blood samples After overnight fasting for $~ 16$ hours, NHPs, which were previously trained to sit consciously and calmly in monkey chair, had $\sim 3 \mathrm{~mL}$ blood samples taken from the cephalic or saphenous vein. The samples were kept in K2 EDTA tubes on ice, and centrifuged at 3,500 rpm for 10 minutes at $4^{\circ} \mathrm{C}$. The resulting plasma was transferred into pre-labeled polypropylene screw-cap vial(s) and stored in a freezer at $-80^{\circ} \mathrm{C}$ until assays were performed.

Urine collection On a different day than blood sampling, the NHPs were housed individually in a stainlesssteel metabolic cage with a net placed between the cage and the urine collection tray (Suzhou Houhuang Animal Laboratory Equipment Technology Co. Ltd, China) to prevent the feces and residual food from contaminating the collected urine. 24-hour urine (10:00AM-10:00AM next day) was collected with the total volume being mixed together, from which $\sim 30 \mathrm{ml}$ urine samples were transferred to a $50 \mathrm{ml}$ tube for centrifugation $\left(4,500 \mathrm{rpm}\right.$ for $10 \mathrm{~min}$ at $\left.4^{\circ} \mathrm{C}\right)$, from which, $\sim 2 \mathrm{ml}$ supernatant was taken for biochemical analysis for specific gravity, $\mathrm{pH}$, ketones, bilirubin, creatinine, cystatin $\mathrm{C}$, glucose, sodium, albumin and protein concentrations by Siemens ADVIA 2400. The 24-hour urinary excretion of total protein (Upro), albumin (Ualb), glucose (Uglu) and creatinine (Ucre) were calculated by the urinary volume (Uvol) and respective concentrations. The ratio of urinary albumin and creatinine (ACR) was calculated by the ratio of urinary concentrations of albumin and creatinine. The estimated glomerular filtration rate (eGFR) was calculated as creatinine clearance with the equation below.

Ucre/Scre $\times 1.73$ / body weight $2 / 3 / 0.118$ 
On the experimental day, NHPs were fasted overnight (16 hours) and received an intramuscular injection of ketamine (Fujian Gutian Pharmaceutical Co. Ltd., Fujian, China) at an initial dose of $10 \mathrm{mg} / \mathrm{kg}$ followed by supplementary doses of $5 \mathrm{mg} / \mathrm{kg}$ as needed. Body temperature was maintained at $\sim 37^{\circ} \mathrm{C}$ by a thermostatically controlled warm water-circulating pad placed underneath the animal. Throughout the experimental period, animals were monitored for vital signs which may trigger intervention (supportive and/or emergent care) and/or study termination if: oxygen saturations $<90 \%$; body temperature $<35{ }^{\circ} \mathrm{C}$; heart rate $<100$ or $>200$ beats/min; respiratory rate $<20$ or $>60 / \mathrm{min}$, serious arrhythmia or respiratory artifacts.

The animals underwent transthoracic echocardiographic scanning with 2-dimentional (2-D) and pulsedwave Doppler mode by a clinical ultrasound instrument (SSD-3500, Hitachi Aloka, Japan). The standard images of the parasternal long axis, apical 3 and 4-chambers were acquired and stored at baseline for measuring cardiac function under resting condition. Dobutamine (Shanghai N0.1 Biochemical \& Pharmaceutical CO. LTD, Lot number: H31021904) at a concentration of $200 \mu \mathrm{g} / \mathrm{mL}$ in physiological saline was then infused into a catheter pre-implanted in the femoral vein at escalating doses of 0 (saline only, for baseline at the resting condition), 5, 10, 20,30, and $40 \mu \mathrm{g} / \mathrm{kg} / \mathrm{min}$ for 7 minutes each dose while the echocardiographic scanning was performed at the plateau of each dose for measuring cardiac functional reserve under dobutamine stress. The mean cardiac output (CO), stroke volume (SV), heart rate $(H R)$, left ventricular (LV) ejection fraction (EF), fraction shortening (FS), end diastolic volume (EDV), early (LVE) and late (LVA) diastolic peak velocity of mitral annulus, velocity-time-integration (VTI LVIT) and $\Delta$ pressure $(\triangle \mathrm{PLVIT})$ of LV inflow trace were captured by the software preinstalled in the ultrasound instrument. Cardiac output reserve (COR) was defined as $\mathrm{CO}$ increment under dobutamine at 40 $\mathrm{mm} / \mathrm{kg} / \mathrm{min}$ from the resting condition at time 0 . After completion of echocardiographic examination, animals were returned to their home cages and closely monitored by well-trained personnel until the animals recovered completely from anesthesia.

\section{$\underline{\text { Statistical analysis }}$}

All the data are expressed as mean \pm standard error (SE). The mean values were compared between control and MetS animals using the Wilcoxon rank-sum test. Correlation between parameters was assessed and visualized using Spearman's rho. Differences for the various cardiac parameters between control and MetS animals were assessed using estimated marginal means obtained from a linear mixed effects model with a random intercept for each individual and dobutamine levels as a factorial covariate. P-values were corrected for multiple testing using Tukey's method. All analyses were performed using the software R 3.6.1, ImerTest and Emmeans packages (R Core Team 2019).

The probability distribution function (PDF) is presented in the supplement data section as the correlogram along with each individual pair of the corresponding correlation coefficient (Figs. S1-6). From the random comparison among all the parameters, several pairs of physiologically meaningful correlation analyses were further conducted using Statistical Analysis System (SAS institute Inc., NC, US) 
to examine the relationships between the prevalence of different relevant parameters with $95 \%$ confidence intervals $(\mathrm{Cl})$ being used to examine the associations in animals with certain risk factors as presented in the results section. A p-value $<0.05$ was used to determine if differences were statistically significant.

\section{Results}

General characterization of dysmetabolic NHPs

A total of 8 normal and 66 NHPs with various degree of dysmetabolism and hyperglycemia were used to measure blood and urine chemistry as well as echocardiography. The correlation analysis was conducted with each pair of the parameters as presented in the supplement Figs. S1-6. Certain pairs of significant correlation were highlighted in Figs S7\&8. The waist circumference (WCU) highly correlated with the degree of obesity measured by total body fat, whilst compromised liver function measured by an elevation of ALT, a liver injury biomarker, highly correlated with higher HbA1c (Fig. S7). Different cardiac functional parameters measured by echocardiography also correlated with each other, e.g., the LV end diastolic volume (EDV), representing diastolic function, positively correlated with LV end systolic volume (ESV), representing systolic function (top), stroke volume (SV) and cardiac output (CO); both representing general cardiac functional performance (Fig. S8). In particular, glycemia not only highly correlated with glucosuria (Fig. 1, top), but also with estimated glomerular filtration rate (eGFR, Fig. 1, bottom).

The characteristics of 8 normal control (CTRL) and 66 Mets NHPs grouped according to 24 hours urinary albumin excretion (Ualb) $\leq$ or $>25 \mathrm{mg}$ are shown in Table 1. As expected, the MetS NHPs with Ualb $>25$ mg were older with hyperglycemic, hyperlipidemic, polyuria, glucosuria, proteinuria with significantly elevated ACR and ALT, as well as 63\% higher eGFR, compared to CTRL. Based on these profiles, 19 MetS with Ualb $>25 \mathrm{mg}$ along with 8 CTRL NHPs were selected for DSE tests.

Cardiac functional reserve following dobutamine stress echocardiography_(DSE).

As shown in Table 2, the basal LV diastolic function measured by E/A ratio was significantly lower in the MetS than CTRL NHPs, while the LV systolic function measured by EF and FS as well as cardiac output (CO) were largely preserved under resting condition. Fig. 2 are the original echocardiography images from a CTRL (top) and a MetS (bottom) NHP, showing that the resting peak LV inflow velocity prior to dobutamine administration was slightly lower in the MetS compared to CTRL NHP, while the LVE was much lower, LVA elevated, hence the E/A ratio increased in the MetS compared to CONT NHP, indicating compromised LV diastolic function under resting condition in MetS NHP. Following DSE with dobutamine infusion at the dose of $40 \mathrm{mg} / \mathrm{kg} / \mathrm{min}$, the increment of LV inflow velocity was markedly smaller in the MetS than CTRL NHP.

Left ventricular diastolic function Under resting condition prior to dobutamine administration, the E/A ratio, a clinical marker of LV diastolic function, was significantly lower in the MetS compared to CTRL NHPs, with significant increase in LVA but not LVE and EDV between the 2 groups (Table 2). Dobutamine 
dose-dependently increased both LVE and LVA, however, the increments were $~ 2.5$ fold smaller in the MetS (LVE: $20 \%$ and LVA: 40\%) than CTRL (LVE: 50\% and LVA: 100\%) NHPs (Fig. 3). Therefore, the E/A ratio dose-dependently decreased in response to dobutamine only in CTRL but not significantly in MetS NHPs, with the peak change $~ 26 \%$ smaller in the MetS $(-12 \pm 3 \%)$ than CTRL $(-38 \pm 5 \%)$ NHPs. There was no significant difference in EDV under resting condition and following dobutamine administration in both groups. Thus, LV filling patterns regarding LVA were evidently compromised, indicating reduced LV diastolic functional in the MetS NHPs.

Left ventricular systolic function There were no significant differences in the resting LV systolic functional indices (EF, FS and ESV) between the MetS and CTRL NHPs (Table 2 and Fig 4). Dobutamine dosedependently increased EF ( $25 \pm 3 \%$ vs. $33 \pm 5 \%)$ and FS ( $44 \pm 5 \%$ vs. $75 \pm 11 \%)$ and decreased ESV $(-44 \pm$ $7 \%$ vs. $-74 \pm 5 \%)$ in both groups, however, with a much smaller degree in the MetS vs. CTRL NHPs.

General cardiac function The resting CO, SV and HR prior to dobutamine infusion were similar between the 2 groups (Table 2 \& Fig. 5). DSE dose-dependently increased all 3 parameters but with a significantly smaller increment in the $\mathrm{CO}$ in the MetS $(63 \pm 8 \%)$ compared to CTRL $(105 \pm 18 \%)$ NHPs following dobutamine infusion at high dose $(40 \mathrm{mg} / \mathrm{kg} / \mathrm{min})$. Thus, the general cardiac function at resting condition was largely preserved but the cardiac output reserve (COR) following dobutamine infusion diminished by $\sim 42 \%(p<0.02)$ in the MetS compared to CTRL NHPs.

\section{Discussion}

Like human diabetic patients, the present data demonstrate that in NHPs with spontaneously developed dysmetabolism, diabetes and proteinuria, echocardiography can detect LV diastolic dysfunction measured by lower E/A ratio, while the LV systolic function measured by EF and FS as well as cardiac output are largely preserved under resting condition. Dobutamine stress echography (DSE) dosedependently enhanced LV functions in both systole and diastole in CTRL and MetS NHPs. However, the MetS NHPs had a $42 \%$ reduction of cardiac output reserve (COR) compared to CTRL NHPs.

The LVE is mainly generated by the LV dilation and LVA by left atrium (LA) contraction. The E/A ratio is commonly used as a clinical marker of LV diastolic function. Under resting condition, LVA was significantly higher indicating an increase in LA contraction, which could partially result from compensatory LA hypotrophy and/or reduced LV distensibility and relaxation capacity as a consequence of cardiomyopathy in the MetS NHPs. As anticipated, infusion of dobutamine dose-dependently enhanced LV dilation measured by elevated LVE as well as LA contraction measured by elevated LVA. However, the degree of the enhancement of LVE and LVA was significantly smaller in the MetS compared to CTRL NHPs. As a result, dobutamine dose-dependently decreased E/A ratio, but the response was much less in the MetS than CTRL NHPs. Thus, DSE elucidated diminished LV diastolic functional reserve in the MetS NHPs with albuminuria.

The basal LV systolic function measured by EF, FS and ESV was largely preserved in the MetS compared to CTRL NHPs under resting condition. However, DSE dose-dependently increased EF and FS and reduced 
ESV with a much smaller response in the MetS than CTRL NHPs. Elevation of ESV represents reduced LV contractility, leading to incapability of pumping adequate blood out of the heart to the circulation at the end of the systolic phase. Thus, MetS NHPs with proteinuria had preserved LV systolic function, but compromised LV systolic functional reserve in response to DSE.

There was no significant difference in the basal CO, SV and HR under resting conditions between the MetS and CTRL NHPs. Dobutamine dose-dependently increased all 3 parameters, but to a lesser degree in the MetS than CTRL NHPs. Therefore, similar to what was seen in the systolic parameters, the MetS NHPs with proteinuria had preserved global cardiac function under rest condition, but DSE unmasked the compromised cardiac output reserve.

Dobutamine is mainly a sympathetic activator with b1-adrenoreceptor (AR) activity on myocytes mediating inotropic effect, b2-AR activity on vascular smooth muscle cells causing vasorelaxation, thus reducing peripheral vascular resistance, and a1-AR activity mediating chronotropic effect [22-24]. It is used in the clinic for decompensated congestive heart failure. In healthy volunteers, dobutamine dosedependently increased cardiac output, which is linearly correlated to its plasma concentrations $[25,26]$. In patients with different forms of cardiac failure, however, the effects of dobutamine are not the same [27]. The inotropic effect of dobutamine increases LV contractility, leading to a decrease in ESV, enabling the heart to pump more blood out from the LV to the circulation with a larger stroke volume. The increases in both the SV and HR contribute to the increase in total cardiac output, which then allows for the baroreceptor mediated response to decrease the systemic vascular resistance, thus maintaining blood pressure homeostasis. With these pharmacological properties of dobutamine in mind, dobutamine stress echocardiography (DSE) does have some limitations in discriminating particular regions of ischemia when multiple ventricular segments are involved [28]. The general performance of the heart is determined by the intrinsic cardiomyocyte contractility, which is also influenced by the preload and afterload of the heart. As mentioned above, dobutamine possesses multiple functions, inotropic effects to stimulate cardiomyocyte contractility, chronotropic effects to increase heart rate, and vasodilatory effects to decrease afterload, all of which are integrated to influence global cardiac performance. Therefore, DSE may not be able to differentiate which exact mechanism contributes to reduced cardiac functional reserve in the MetS NHPs. Regardless of this limitation, the independence of DSE from the effects of LV hypertrophy and hypertension is still used in patients with renal disease [29] to document resting LV function as well as it's functional reserve, both of which are prognostically important factors in end-stage renal failure [30].

Cardiorenal dysfunctions are common diabetic complications on both end-organs that may interact with each other. Heart failure with reduced ejection fraction (HFrEF) causes renal dysfunction via kidney hypoperfusion; conversely, renal impairment is observed in a great number of heart failure patients with preserved ejection fraction (HFpEF) and is associated with an increased risk of mortality via uremic toxins, increased fluid afterload, etc. [31-35]. Combined heart and kidney failure in patients pose several clinical challenges, including diagnostic difficulties and therapeutic dilemmas, since some heart failure medications may cause, or are contraindicated, in the presence of renal failure [38]. 
Indeed, the present NHP model with metabolic disorders and diabetes also accompanied with significant proteinuria, is similar to diabetic patients with cardiorenal complications. Proteinuria is an early sign and sensitive biomarker for diabetic nephropathy [36-38], which is strongly correlated with increased risk of cardiovascular and cerebrovascular diseases $[33,39,40]$. In patients with metabolic disorders and diabetes, the initial histopathology change is tubular injury that leads to protein leakage in early stages of diabetic nephropathy before the global glomerular filtration function is compromised [41, 42]. Instead, hyperglycemia could even change kidney hemodynamics, leading to glomerular hyperfiltration in early stages of diabetes which further contributes to the progression of kidney injury [43]. Indeed, in the present study, the eGFR in the MetS NHPs with proteinuria was $~ 50 \%$ higher than that in CTRL, indicating the MetS NHPs used in this study are still at an early stage of diabetic nephropathy with kidney function at the hyperfiltration stage without significant increase in BUN and serum creatinine.

\section{Conclusion}

This study demonstrates that similar to that observed in human diabetic patients with albuminuria, NHPs with spontaneously developed dysmetabolism, diabetes and proteinuria show compromised diastolic function and preserved LV systolic function under resting condition. Dobutamine dose-dependently enhanced LV function in both systole and diastole and cardiac output, however, to a much lesser degree in the MetS than CTRL NHPs, indicating a compromised cardiac functional reserve, demonstrating the importance of stress tests to fully elucidate the disease phenotype and to capture treatment effects in intervention studies. Since NHPs have phylogenetic proximity to humans, the NHP model of dysmetabolism and diabetes with cardiorenal complications, along with the established DSE protocol, can be used as a model with expected high translational value for preclinical researches in testing and profiling the safety and efficacy of novel therapeutics.

\section{Abbreviations}

a/b-ARs: Alpha/beta-adrenergic receptors

ACR: Albumin creatinine ratio

CO: Cardiac output

COR: Cardiac output reserve

CTRL: Normal control

DSE: Dobutamine stress echocardiography

E/A: LVE/LVA ratio

EDV/ESV: LV end diastolic/systolic volume 
EF/FS: LV ejection fraction/fractional shortening

eGFR: Estimated glomerular filtration rate

HR: Heart rate

LA/LV: Left atrium/Left ventricle

LVE/LVA: LV early/late trans-mitral Doppler inflow velocity

MetS: Nonhuman primate with metabolic syndrome

NHP: Nonhuman primate

SV: Stroke volume

WCU: Waist circumference

\section{Declarations}

Ethics approval and consent to participate

All experimental protocols and procedures were approved by Crown Bioscience's institutional animal care and use committee (IACUC).

Consent for publication

All the co-authors have carefully read and approved the final version of the manuscript for submission to Journal of Translational Medicine for publication.

Availability of data and materials

All the experimental data will be available upon request.

Competing interests

All the authors were the employees of AstraZeneca or Crown Bioscience Inc. and declare no conflict of interests in this study.

\section{Funding}

Not applicable.

Authors' contributions

JW, YQL, LMG, YXW took charge of the study design, data analysis, result interpretation, discussion and manuscript writing, had full access to all the data in the study and are responsible for the integrity and 
accuracy of the experimental procedures and data analysis. YQL and YQJ performed echocardiography experiment, blood and urine sampling and bioanalysis. BCM, CZ and PK performed statistical analysis. $\mathrm{CW}$ and YFX involved in the study discussion.

\section{Acknowledgements}

Part of the data in this manuscript have been presented at the 2018 American Heart Association annual conference. The authors gratefully acknowledge the excellent technical assistance of Crown Bioscience animal center, and other vivarium staff for their professional care of the NHPs and assistance in the experiments. We also appreciate Dr. X. Gao for providing guidance on echocardiography techniques.

\section{References}

1. Scherrer-Crosbie M, Steudel W, Ullrich R, Hunziker PR, Liel-Cohen N, Newell J, Zaroff J, Zapol WM, Picard $\mathrm{MH}$ : Echocardiographic determination of risk area size in a murine model of myocardial ischemia. Am J Physiol 1999, 277(3):H986-992.

2. Bell DS: Heart failure: the frequent, forgotten, and often fatal complication of diabetes. Diabetes Care 2003, 26(8):2433-2441.

3. Sharir T, Germano G, Kavanagh PB, Lai S, Cohen I, Lewin HC, Friedman JD, Zellweger MJ, Berman DS: Incremental prognostic value of post-stress left ventricular ejection fraction and volume by gated myocardial perfusion single photon emission computed tomography. Circulation 1999, 100(10):1035-1042.

4. Matyal R, Skubas NJ, Shernan SK, Mahmood F: Perioperative assessment of diastolic dysfunction. Anesth Analg 2011, 113(3):449-472.

5. Nagueh SF, Appleton CP, Gillebert TC, Marino PN, Oh JK, Smiseth OA, Waggoner AD, Flachskampf FA, Pellikka PA, Evangelista A: Recommendations for the evaluation of left ventricular diastolic function by echocardiography. J Am Soc Echocardiogr 2009, 22(2):107-133.

6. Pearce K, Chambers J: Safety and efficacy of physiologist-led dobutamine stress echocardiography. Echo Res Pract 2018, 5(4):E9-E10.

7. Abdel-Salam Z, Nammas W: Predictors of viability in patients with negative low-dose dobutamine stress echocardiograph. Arq Bras Cardio/ 2011, 96(3):188-195.

8. Jin Y, Lin W, Becher H, Choy JB, Xia S: Safety of Single-Line Infusion of Dobutamine and UltrasoundEnhancing Agents during Stress Echocardiography. J Am Soc Echocardiogr 2018, 32(3):423-425.

9. Sato K, Sankaramangalam K, Kandregula K, Bullen JA, Kapadia SR, Krishnaswamy A, Mick S, Rodriguez LL, Grimm RA, Menon V et al: Contemporary Outcomes in Low-Gradient Aortic Stenosis Patients Who Underwent Dobutamine Stress Echocardiography. J Am Heart Assoc 2019, 8(6):e011168.

10. Gupta P, Chockalingam A: Dobutamine stress echocardiogram-related in-stent thrombosis with acute myocardial infarction. BMJ Case Rep 2019, 12(1). 
11. Nemchyna O, Gebker R, Schoenrath F, Knosalla C, Falk V, Knierim J: Longitudinal strain under dobutamine stress echocardiography in patient with light chain cardiac amyloidosis. Eur Heart $J$ Cardiovasc Imaging 2018, 20(4):486.

12. Baloglu I, Turkmen K, Selcuk NY, Tonbul HZ, Ozcicek A, Hamur H, lyisoy S, Akbas EM: The Relationship Between Visceral Adiposity Index and Epicardial Adipose Tissue in Patients with Type 2 Diabetes Mellitus. Exp Clin Endocrinol Diabetes 2019, 5(6).

13. Samiei N, Parsaee M, Pourafkari L, Tajlil A, Pasbani Y, Rafati A, Nader ND: The value of negative stress echocardiography in predicting cardiovascular events among adults with no known coronary disease. J Cardiovasc Thorac Res 2019, 11(2):85-94.

14. Wang BD, Sun GF, Liu YQ, Wang H, Wang XL, Chng K, Qiao W, Wang Y-X, Xiao Y-F: Dysglycemia and Dyslipidemia Models in Nonhuman Primates: Part II Model of Naturally Occurring and Experimental models of Obesity. J Diabetes Metab 2016 7(1).

15. Wang XL, Wang BD, Sun GF, Wu J, Liu YQ, Wang Y-X, Xiao Y-F: Dysglycemia and Dyslipidemia Models in Nonhuman Primates: Part I Model of Naturally Occurring Diabetes. J Diabetes Metab 2015, S13:

16. Liu YQ, Gao JJ, Wang XL, Wang Y-X, Xiao Y-F: Dysglycemia and Dyslipidemia Models in Nonhuman Primates: Part III. Type I or II Diabetogenic Effects of Streptozocin J Diabetes Metab 2019, 10(3).

17. Guo S, Qian WB, Du FL, Wang BD, Wang XL, Fang YP, Chen X, Benzinou M, Gregoire FM, Staup MP et al: Proteinuria in Cynomolgus macaques (Macaca fascicularis) with Spontaneously Developed Metabolic Disorder and Diabetes: Transcriptome Analysis of Biopsy Kidney. J Diabetes \& Metabolism 2014, 5(2).

18. Liu YQ, Gu HH, Wang H, Wang BD, Wang XL, Aoyagi G, Xiao Y-F, Chng K, Gao X, Wang JH et al: Hepatic Steatosis and Fibrosis in Obese, Dysmetabolic and Diabetic Nonhuman Primates Quantified by Noninvasive Echography. Journal of Diabetes and Metabolism 2017, 8(10).

19. Gu HH, Liu YQ, Mei S, Wang BD, Sun GF, Wang XL, Xiao Y-F, Staup MP, Gregoire FM, Chng K et al: Left ventricular diastolic dysfunction in nonhuman primate model of dysmetabolism and diabetes. $B M C$ Cardiovasc Disord 2015, 15:141.

20. Vincelette J, Martin-McNulty B, Vergona R, Sullivan ME, Wang Y-X: Reduced cardiac functional reserve in apolipoprotein E knockout mice Transl Res 2006, 148:30-36.

21. Wang Y-X, Fitch R, Li W, Werner M, Halks-Miller M, Lillis B, Vergona R, Post J, Sullivan M, Verhallen P: Reduction of cardiac functional reserve and elevation of aortic stiffness in hyperlipidemic Yucatan minipigs with systemic and coronary atherosclerosis Vascular Pharmacol 2002, 39:69-76.

22. Kislitsina ON, Rich JD, Wilcox JE, Pham DT, Churyla A, Vorovich EB, Ghafourian K, Yancy CW: ShockClassification and Pathophysiological Principles of Therapeutics. Curr Cardiol Rev 2018, 15(2):102113.

23. Ruf K, Wirbelauer J, Beissert A, Frieauff E: Successful treatment of severe arterial hypotension and anuria in a preterm infant with renal tubular dysgenesis- a case report. Matern Health Neonatol Perinatol 2019, 4:27. 
24. Basile J, Egan B, Punzi H, Ali S, Li Q, Patel M, Neutel J: Risk of Hospitalization for Cardiovascular Events with beta-Blockers in Hypertensive Patients: A Retrospective Cohort Study. Cardiol Ther 2018, 7(2):173-183.

25. Ahonen J, Aranko K, livanainen A, Maunuksela EL, Paloheimo M, Olkkola KT: Pharmacokineticpharmacodynamic relationship of dobutamine and heart rate, stroke volume and cardiac output in healthy volunteers. Clin Drug Investig 2008, 28(2):121-127.

26. Fowler MB, Timmis AD, Crick JP, Vincent R, Chamberlain DA: Comparison of haemodynamic responses to dobutamine and salbutamol in cardiogenic shock after acute myocardial infarction. $\mathrm{Br}$ Med J (Clin Res Ed) 1982, 284(6309):73-76.

27. Beregovich J, Bianchi C, D'Angelo R, Diaz R, Rubler S: Haemodynamic effects of a new inotropic agent (dobutamine) in chronic cardiac failure. Br Heart J 1975, 37(6):629-634.

28. John D, Stoner I, James L, Belon, Donald CH: Comparison of dobutamine and dopamine in treatment of severe heart failure1. British Heart Journal 1977, 39:536-539.

29. Usher BW, Jr., O'Brien TX: Recent advances in dobutamine stress echocardiography. Clin Cardiol 2000, 23(8):560-570.

30. Marwick TH, Lauer MS, Lobo A, Nally J, Braun W: Use of dobutamine echocardiography for cardiac risk stratification of patients with chronic renal failure. J Intern Med 1998, 244(2):155-161.

31. Santoro A: Heart failure and cardiorenal syndrome in the elderly. J Nephrol 2012, 25(Supple 19):S6772.

32. Vinod P, Krishnappa V, Chauvin AM, Khare A, Raina R: Cardiorenal Syndrome: Role of Arginine Vasopressin and Vaptans in Heart Failure. Cardiol Res 2017, 8(3):87-95.

33. Jindal A, Garcia-Touza M, Jindal N, Whaley-Connell A, Sowers JR: Diabetic kidney disease and the cardiorenal syndrome: old disease, new perspectives. Endocrinol Metab Clin North Am 2013, 42(4):789-808.

34. Ter Maaten JM, Damman K, Verhaar MC, Paulus WJ, Duncker DJ, Cheng C, van Heerebeek L, Hillege $\mathrm{HL}$, Lam CS, Navis G et al: Connecting heart failure with preserved ejection fraction and renal dysfunction: the role of endothelial dysfunction and inflammation. Eur J Heart Fail 2016, 18(6):588598.

35. Ter Maaten JM, Voors AA: Renal dysfunction in heart failure with a preserved ejection fraction: cause or consequence? Eur J Heart Fail 2015, 18(1):113-114.

36. Colhoun HM, Marcovecchio ML: Biomarkers of diabetic kidney disease. Diabetologia 2018, 61(5):996-1011.

37. Hickson LJ, Balls-Berry JE, Jaffe AS, Rule AD: Biomarkers Associated with Progression of Diabetic Kidney Disease: Do They Hold the Same Meaning for Blacks and Women?J Am Soc Nephrol 2018, 29(6):1781.

38. Zheng S, Powell DW, Zheng F, Kantharidis P, Gnudi L: Diabetic Nephropathy: Proteinuria, Inflammation, and Fibrosis. J Diabetes Res 2016, 2016:5241549. 
39. Rabizadeh S, Nakhjavani M, Esteghamati A: Cardiovascular and Renal Benefits of SGLT2 Inhibitors: A Narrative Review. Int J Endocrinol Metab 2019, 17(2):e84353.

40. Barrett EJ, Liu Z, Khamaisi M, King GL, Klein R, Klein BEK, Hughes TM, Craft S, Freedman BI, Bowden DW et al: Diabetic Microvascular Disease: An Endocrine Society Scientific Statement. J Clin Endocrinol Metab 2017, 102(12):4343-4410.

41. Chagnac A ZB, Rozen-Zvi B, Herman-Edelstein M: Consequences of Glomerular Hyperfiltration: The Role of Physical Forces in the Pathogenesis of Chronic Kidney Disease in Diabetes and Obesity. Nephron 2019, 4:1-5.

42. Gonçalves-Dias C MJ, Correia MJ, Coelho NR, Antunes AMM, Macedo MP, Monteiro EC, Soto K, Pereira SA.: Mercapturate Pathway in the Tubulocentric Perspective of Diabetic Kidney Disease. Nephron 2019, Jan 9:1-7.

43. Wang Y-X, Brooks D, Edwards R: Attenuated glomerular cGMP stimulation and renal vasodilatation in streptozotocin-induced diabetic rats. Am J Physio/ 1993, 264(Regulatory Integrative Comp Physiol 33):R952-R956.

\section{Tables}

Table 1. Blood and urine chemistry measurements in the normal (CTRL) and dysmetabolic (MetS) NHPs

Data presented as Mean \pm SE 


\begin{tabular}{|c|c|c|c|c|}
\hline Parameters & Control & $\begin{array}{c}\text { MetS } 1 \\
\text { Ualb } \leqslant 25 \\
\text { mg/24 hr. }\end{array}$ & $\begin{array}{c}\text { MetS } 2 \\
\text { Ualb }>25 \\
\mathrm{mg} / \mathbf{2 4} \mathrm{hr} .\end{array}$ & $\begin{array}{c}\text { Control vs } \\
\text { MetS } 2 \\
\text { p value } \\
\end{array}$ \\
\hline Number of NHPs & 8 & 47 & 19 & \\
\hline Age (year) & $12 \pm 0.6$ & $16 \pm 0.4$ & $17 \pm 1$ & 0.003 \\
\hline Body weight (kg) & $11 \pm 0.5$ & $9 \pm 0.4$ & $10 \pm 0.7$ & 0.630 \\
\hline \multicolumn{5}{|l|}{ Blood chemistry } \\
\hline Glucose (mg/dL) & $61 \pm 3$ & $157 \pm 16$ & $178 \pm 18$ & 0.000 \\
\hline Insulin $(\mathrm{mIU} / \mathrm{L})$ & $64 \pm 24$ & $116 \pm 23$ & $90 \pm 26$ & 0.980 \\
\hline $\mathrm{TG}(\mathrm{mg} / \mathrm{dL})$ & $62 \pm 11$ & $212 \pm 34$ & $321 \pm 92$ & 0.052 \\
\hline $\mathrm{TC}(\mathrm{mg} / \mathrm{dL})$ & $91 \pm 9$ & $137 \pm 10$ & $150 \pm 22$ & 0.009 \\
\hline $\mathrm{HDL}(\mathrm{mg} / \mathrm{dL})$ & $43 \pm 6$ & $45 \pm 3$ & $41 \pm 5$ & 0.870 \\
\hline $\mathrm{LDL}(\mathrm{mg} / \mathrm{dL})$ & $28 \pm 3$ & $68 \pm 7$ & $75 \pm 16$ & 0.012 \\
\hline $\operatorname{ALT}(\mathrm{U} / \mathrm{L})$ & $22 \pm 5$ & $117 \pm 16$ & $69 \pm 9$ & 0.001 \\
\hline $\operatorname{AST}(\mathrm{U} / \mathrm{L})$ & $28 \pm 3$ & $41 \pm 2$ & $33 \pm 2$ & 0.190 \\
\hline $\mathrm{BUN}(\mathrm{mmol} / \mathrm{L})$ & $5 \pm 0.2$ & $6 \pm 0.2$ & $10 \pm 3$ & 0.160 \\
\hline Creatinine $(\mu \mathrm{mol} / \mathrm{L})$ & $81 \pm 5$ & $70 \pm 4$ & $112 \pm 51$ & 0.075 \\
\hline \multicolumn{5}{|l|}{ Urine analysis } \\
\hline eGFR $(\mathrm{ml} / \mathrm{min}$ per $1.73 \mathrm{~m} 2)$ & $128 \pm 10$ & $163 \pm 11$ & $209 \pm 32$ & 0.094 \\
\hline $\operatorname{ACR}(24 \mathrm{hr})$ & $1 \pm 0.4$ & $9 \pm 1.3$ & $134 \pm 33$ & 0.000 \\
\hline Urine protein (mg/24hr) & $17 \pm 1.6$ & $15 \pm 2.3$ & $225 \pm 59$ & 0.001 \\
\hline Urine albumin (mg/24hr) & $3 \pm 0.5$ & $8 \pm 0.8$ & $156 \pm 47$ & 0.000 \\
\hline Urine nitrogen (mmol/24hr) & $54 \pm 6.3$ & $57 \pm 3.4$ & $76 \pm 8.1$ & 0.067 \\
\hline Urine creatinine $(\mathrm{mmol} / 24 \mathrm{hr})$ & $2 \pm 0.3$ & $1 \pm 0.1$ & $2 \pm 0.3$ & 0.075 \\
\hline Urine glucose (mmol/24hr) & $16 \pm 11$ & $79 \pm 13$ & $125 \pm 30$ & 0.006 \\
\hline Urine volume (ml/24hr) & $274 \pm 43$ & $545 \pm 50$ & $592 \pm 78$ & 0.044 \\
\hline
\end{tabular}

Table 2. Resting cardiac functional measurements in the normal (CTRL) and dysmetabolic (MetS) NHPs

Data presented as Mean \pm SE 


\begin{tabular}{|c|c|c|c|c|}
\hline Parameters & Control & $\begin{array}{c}\text { MetS 1 } \\
\text { Ua lb }{ }_{6} / x_{2} 25 \\
\text { mg/24 hr. }\end{array}$ & $\begin{array}{c}\text { MetS } 2 \\
\text { Ualb }>25 \\
\text { mg/24 hr. }\end{array}$ & $\begin{array}{c}\text { Control vs } \\
\text { Mets } 2 \\
\text { p value } \\
\end{array}$ \\
\hline Number of NHPs & 8 & 47 & 19 & \\
\hline Age (year) & $12 \pm 0.6$ & $16 \pm 0.4$ & $17 \pm 1$ & 0.003 \\
\hline Body weight (kg) & $11 \pm 0.5$ & $9 \pm 0.4$ & $10 \pm 0.7$ & 0.630 \\
\hline \multicolumn{5}{|l|}{ Cardiac function } \\
\hline \multicolumn{5}{|c|}{ Left Ventricular diastolic function } \\
\hline E/A ratio & $1.5 \pm 0.13$ & $1.0 \pm 0.03$ & $1.0 \pm 0.06$ & 0.003 \\
\hline $\operatorname{LVE}(\mathrm{m} / \mathrm{s})$ & $0.8 \pm 0.03$ & $0.6 \pm 0.06$ & $0.6 \pm 0.04$ & 0.074 \\
\hline LVA $(\mathrm{m} / \mathrm{s})$ & $0.5 \pm 0.04$ & $0.7 \pm 0.03$ & $0.6 \pm 0.03$ & 0.049 \\
\hline EDV $(\mathrm{ml})$ & $18 \pm 3$ & $16 \pm 1$ & $19 \pm 2$ & 0.920 \\
\hline \multicolumn{5}{|c|}{ Left V entricular systolic function } \\
\hline EF (\%) & $71 \pm 3$ & $67 \pm 1$ & $65 \pm 2$ & 0.075 \\
\hline FS (\%) & $38 \pm 2$ & $35 \pm 1$ & $34 \pm 1$ & 0.067 \\
\hline $\mathrm{ESV}(\mathrm{ml})$ & $5 \pm 0.8$ & $6 \pm 0.4$ & $7 \pm 1.1$ & 0.350 \\
\hline \multicolumn{5}{|l|}{ General } \\
\hline $\mathrm{CO}(\mathrm{L} / \mathrm{min})$ & $1.7 \pm 0.3$ & $1.6 \pm 0.1$ & $1.6 \pm 0.1$ & 0.870 \\
\hline SV (ml) & $12 \pm 2$ & $11 \pm 1$ & $12 \pm 1$ & 0.830 \\
\hline HR (BPM) & $138 \pm 7$ & $150 \pm 3$ & $140 \pm 4$ & 0.820 \\
\hline \multicolumn{5}{|l|}{ Others } \\
\hline VTI LVOT (cm) & $11 \pm 0.5$ & $12 \pm 0.5$ & $11 \pm 0.5$ & 0.960 \\
\hline$\triangle \mathrm{PLVOT}(\mathrm{mmHg})$ & $2 \pm 0.24$ & $3 \pm 0.27$ & $2 \pm 0.23$ & 0.490 \\
\hline
\end{tabular}

Figures 

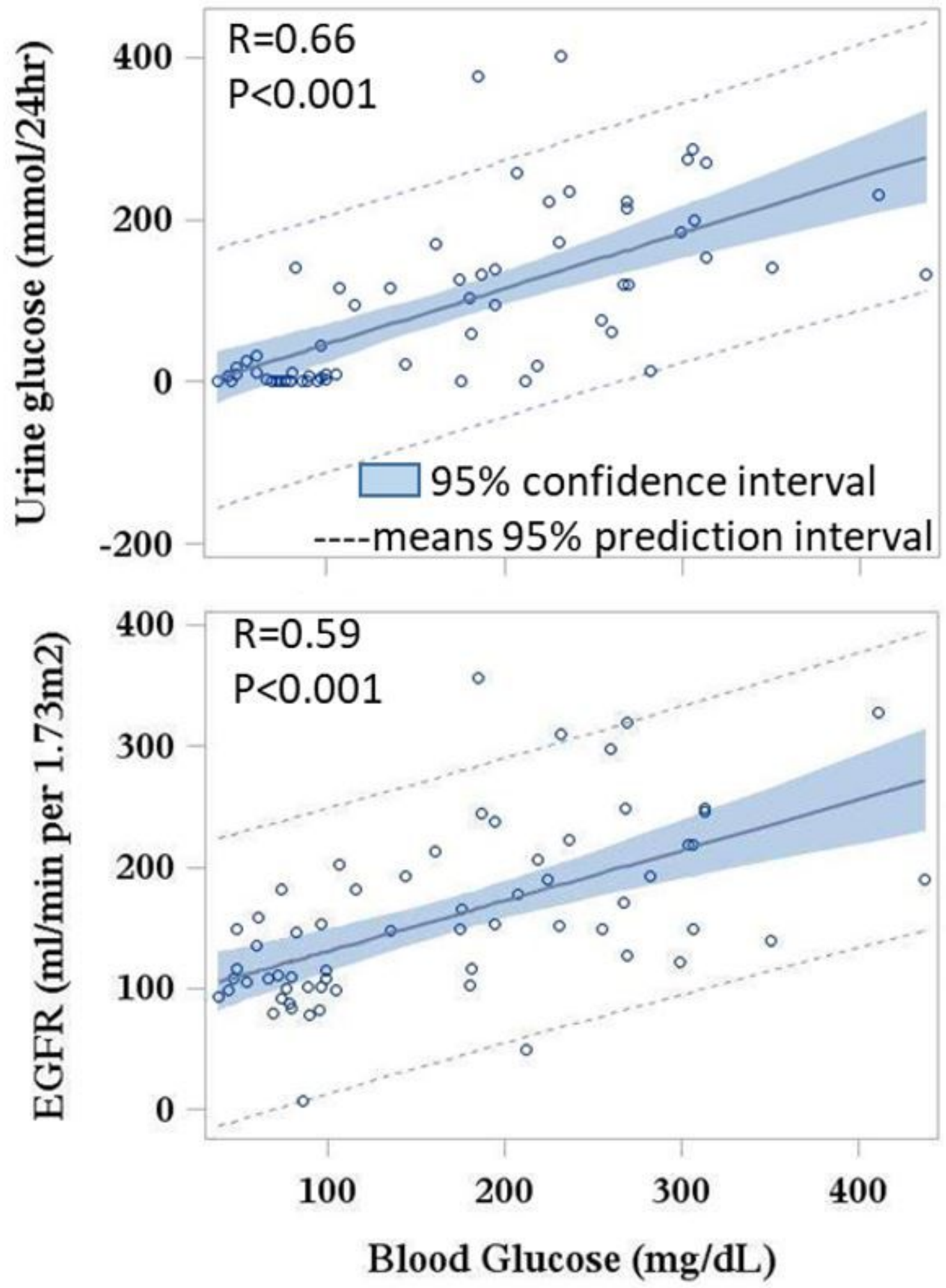

Figure 1

Correlation of blood glucose concentration vs. urinary glucose excretion (top); or estimated glomerular filtration rate (eGFR, bottom) in 8 normal control NHPs and 66 dysmetabolic NHPs with different degree of proteinuria 


\section{Resting \\ Dobutamine \\ Condition \\ $(40 \mu \mathrm{g} / \mathrm{kg} / \mathrm{min})$}

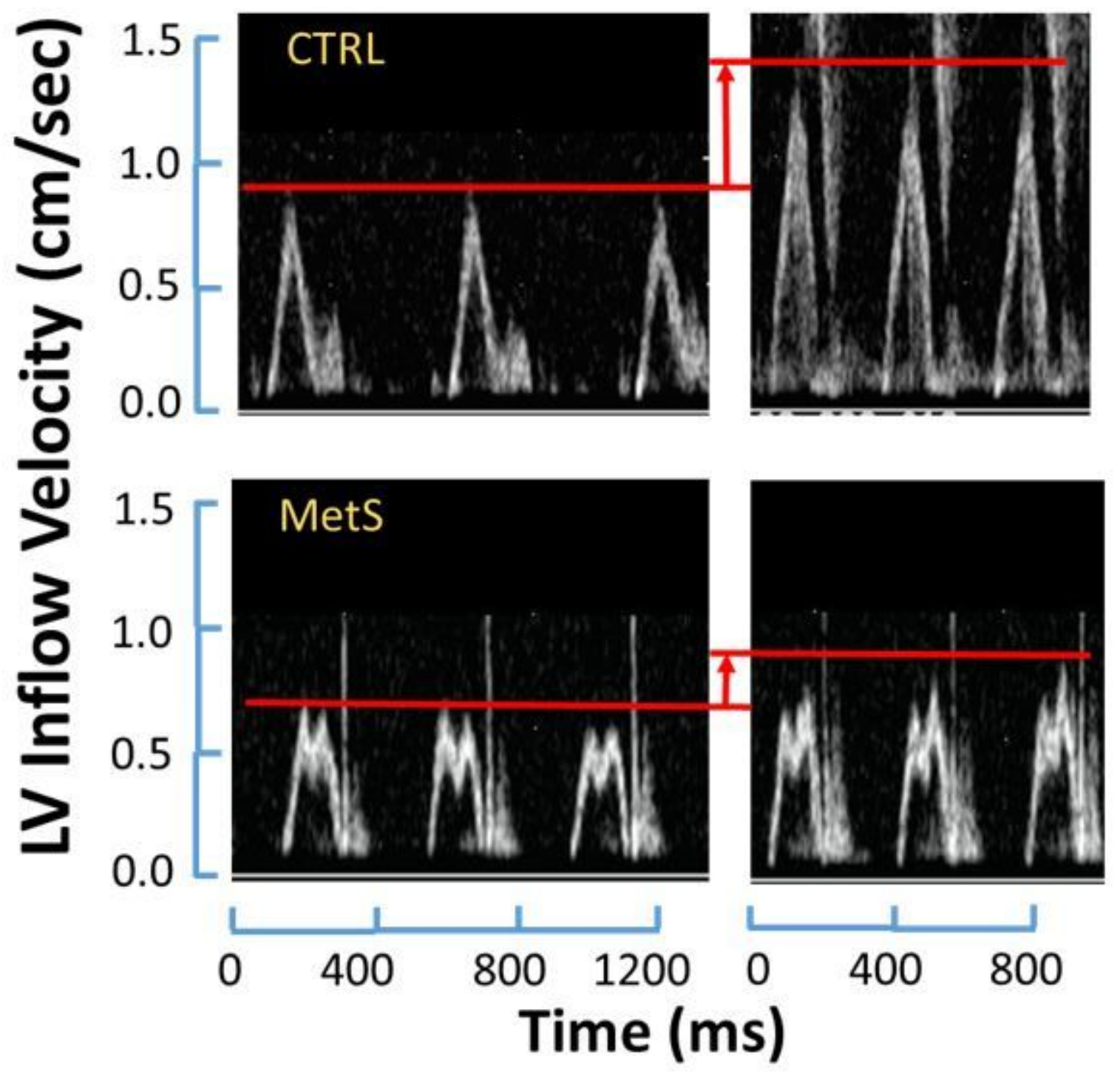

Figure 2

Left ventricle inflow velocity at resting condition before (left) and after (right) dobutamine infusion at the dose of $40 \square \mathrm{g} / \mathrm{kg} / \mathrm{min}$ in a representative normal control NHP (CTRL, top) and dysmetabolic NHP with proteinuria (MetS, bottom) The horizontal line represents the average peak level of LV inflow, and the vertical arrow is the increment of in LV inflow following dobutamine stress echocardiography. 


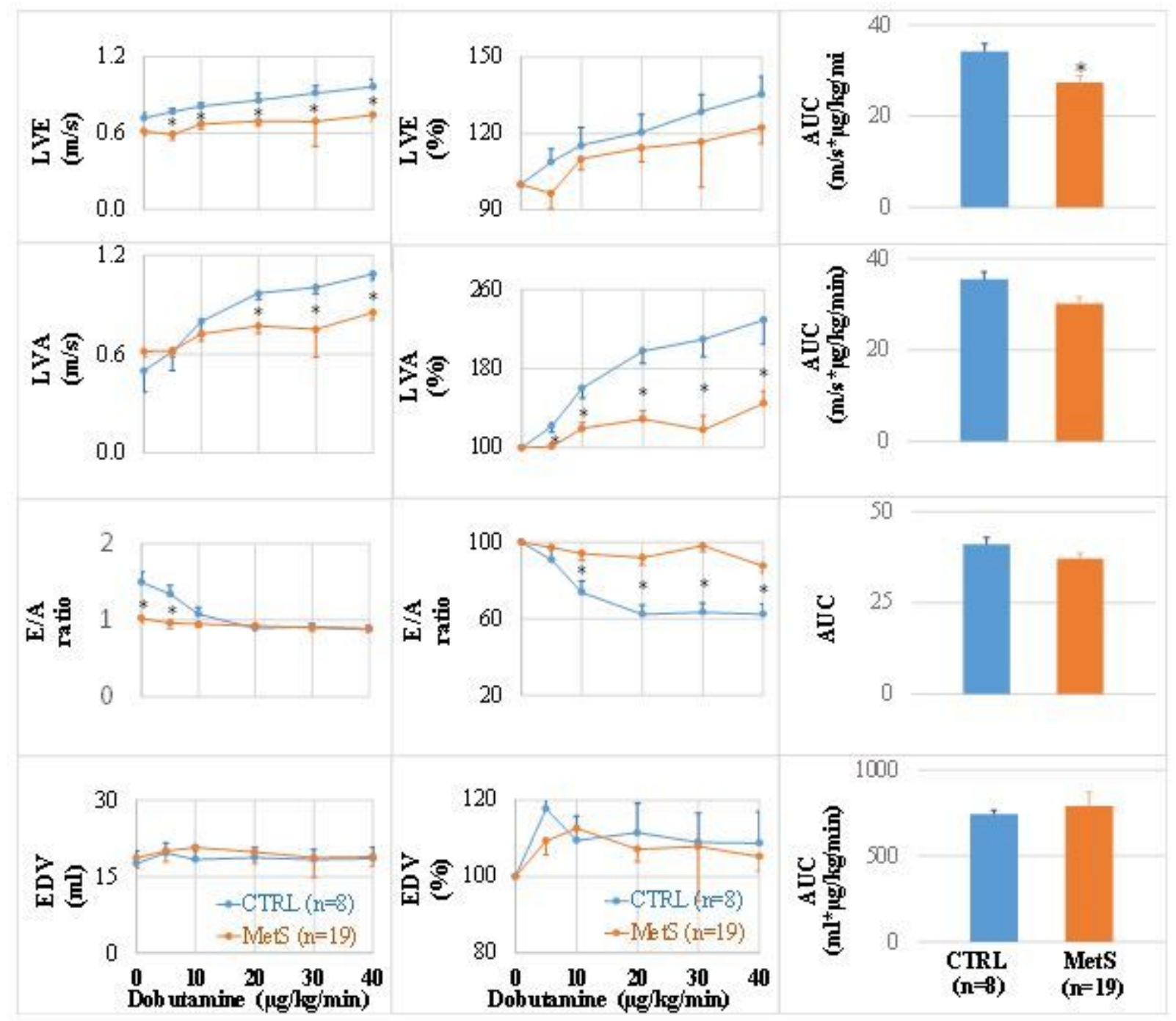

Figure 3

Left ventricular diastolic functional reserve following dobutamine stress echocardiography (DSE) in the normal NHPs and (CTRL) and dysmetabolic NHPs with proteinuria (MetS) Left: Dose-response curves of left ventricular early (LVE) and late (LVA) filling velocity; LVE/LVA ratio (E/A); and end diastolic volume (EDV). Middle: Percent changes of the dose-response curves. Right: Area under the dose-response curves. *, $p<0.05$ between the 2 groups at the corresponding time points by t-test. Data presented as Mean $+\mathrm{SE}$ 


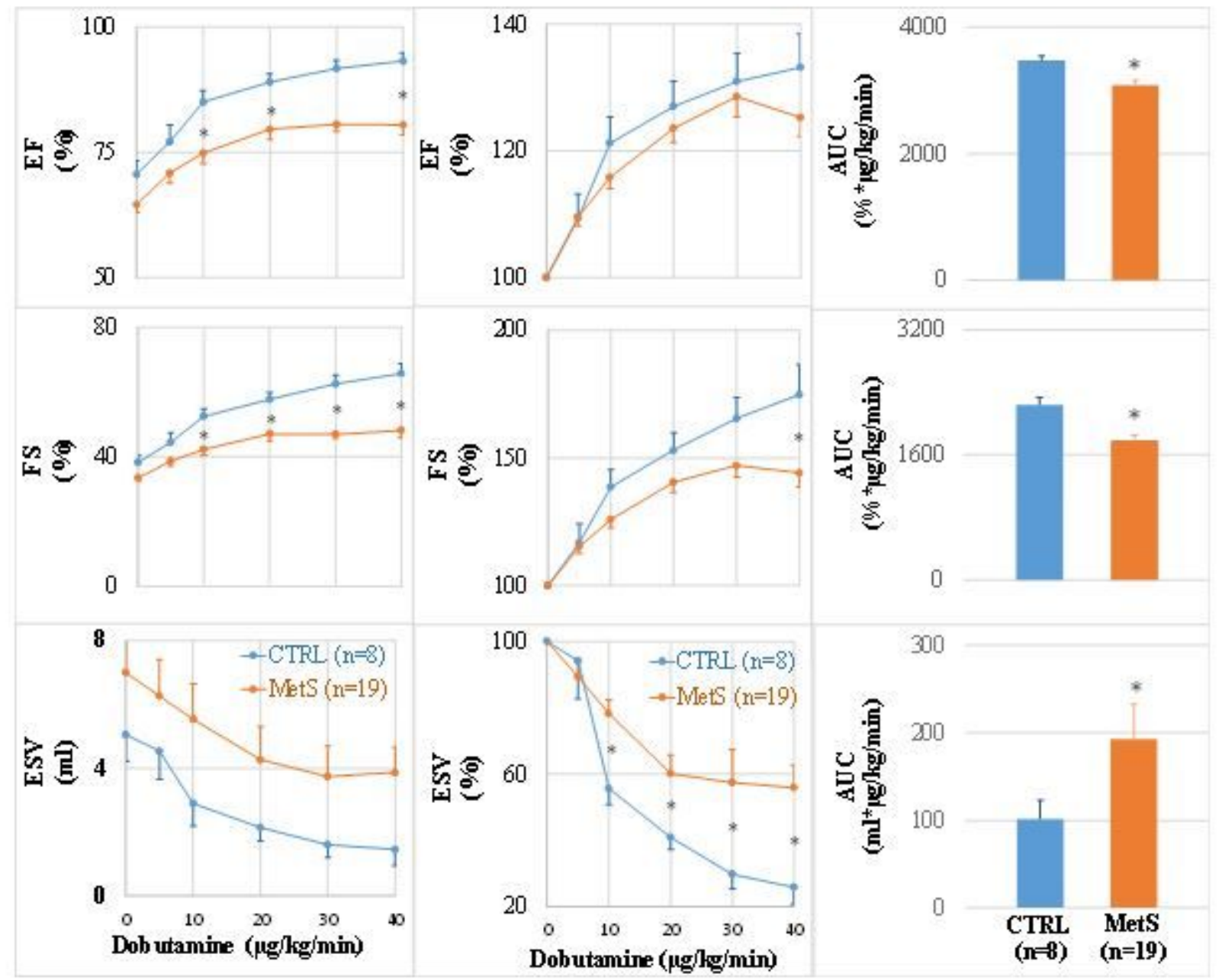

Figure 4

Left ventricular systolic functional reserve following dobutamine stress echocardiography (DSE) in the normal NHPs (CTRL) and dysmetabolic NHPs with proteinuria (MetS) Left: Dose-response curves of left ventricular ejection fraction (EF), fractional shortening (FS), and end systolic volume (ESV). Middle: Percent changes of the dose-response curves. Right: Area under the dose-response curves. ${ }^{*}, \mathrm{p}<0.05$ between the 2 groups at the corresponding time points by t-test. Data presented as Mean $+\mathrm{SE}$ 


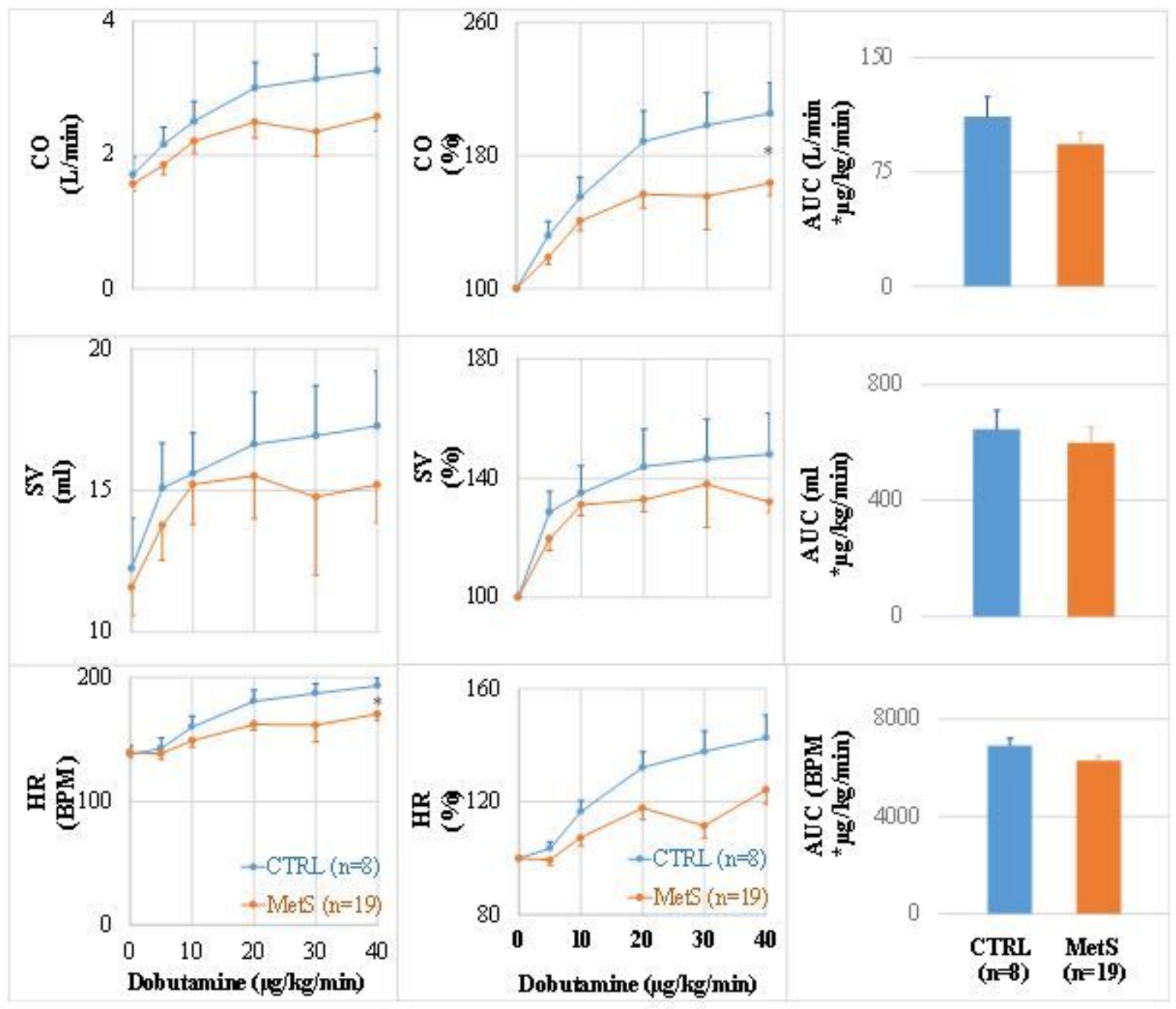

Figure 5

General cardiac functional reserve following dobutamine stress echocardiography (DSE) in the normal NHPs (CTRL) and dysmetabolic NHPs with proteinuria (MetS) Left: Dose-response curves of cardiac output (CO), stroke volume (SV) and heart rate (HR). Middle: Percent changes of the dose-response curves. Right: Area under the dose-response curves. ${ }^{*}, p<0.05$ between the 2 groups at the corresponding time points by t-test. Data presented as Mean $+\mathrm{SE}$

\section{Supplementary Files}

This is a list of supplementary files associated with this preprint. Click to download.

- Supplementaldata.docx 